\title{
Identification of the NLS and NES motifs of VP2 from chicken anemia virus and the interaction of VP2 with mini-chromosome maintenance protein 3
}

Jai-Hong Cheng ${ }^{1 \dagger}$, Shyang-Chwen Sheu ${ }^{2+}$, Yi-Yang Lien ${ }^{3}$, Meng-Shiunn Lee ${ }^{4}$, His-Jien Chen ${ }^{5}$, Wen-Hong Su ${ }^{1}$ and Meng-Shiou Lee 6* $^{*}$

\begin{abstract}
Background: VP2 of chicken anemia virus (CAV) is a dual-specificity phosphatase required for virus infection, assembly and replication. The functions of the nuclear localization signal (NLS) and nuclear export signal (NES) of VP2 in the cell, however, are poorly understood. Our study identified the presence of a NLS in VP2 and showed that the protein interacted significantly with mini-chromosome maintenance protein 3 (MCM3) in the cell.

Results: An arginine-lysine rich NLS could be predicted by software and spanned from amino acids 133 to 138 of VP2. The critical amino acids residues between positions 136 and 138, and either residue 133 or 134 are important for nuclear import in mammalian cells based on systematic mutagenesis. A NES is also predicted in VP2; however the results suggest that no functional NES is present and that this protein is CRM1 independent. It was also shown that VP2 is a chromatin binding protein and, notably, using a co-immunoprecipitation assay, it was found that VP2 association with MCM3 and that this interaction does not require DSP activity.
\end{abstract}

Conclusions: VP2 contains a NLS that span from amino acids 133 to 138. VP2 is a CRM1 independent protein during nuclear export and associates with MCM3 in cells.

\section{Background}

CAV is a small non-enveloped, single-stranded, circular DNA virus and was first isolated in Japan [1]. This virus belongs to the genus Gyrovirus of the Circoviridae family and causes a severe immunosuppressive syndrome and anemia in chickens [2]. It is a ubiquitous pathogen of chickens and has a worldwide distribution. According to epidemiological studies, it has been shown that almost all newly hatched chicks are susceptible to $\mathrm{CAV}$ as a clinical syndrome, but not mature chickens [3]. Normally, young chickens, generally fewer than 2 weeks of age, are very susceptible to this virus through vertical transmission via hatching eggs [4]. The virus typically induces aplasia of the bone marrow and

\footnotetext{
* Correspondence: leemengshiou@mail.cmu.edu.tw

† Contributed equally

${ }^{6}$ School of Chinese Pharmaceutical Science and Chinese Medicine Resources,

China Medical University, Taichung, Taiwan

Full list of author information is available at the end of the article
}

damage to lymphoid tissue, which causes anemia and acute immunodeficiency syndrome $[5,6]$. Up to the present, a large number of isolates, including strains from Australia, Bangladesh, Brazil, China, Germany, Malaysia, Nigeria, Slovenia, Taiwan and USA, have been reported and have had full or partial sequences published [7-9].

The DNA genome of CAV is about $2.3 \mathrm{~kb}$ in size [10-12] and there are three ORFs present on the negative sense genome. At least three proteins are produced from a single polycistronic $2.1 \mathrm{~kb}$ mRNA that is reliable produced as a single molecule and contains a promoter, TATA-box, and poly (A) signal $[11,13,14]$. The three translated proteins are called VP1, VP2 and VP3. VP1 is a $51 \mathrm{kDa}$ protein that is the structure protein involved in assembly of the viral caspid [15]. VP2 is a $24 \mathrm{kDa}$ protein that contains a dual-specificity phosphatase (DSP) activity and some apoptotic activity $[2,16,17]$. However, VP2's apoptotic activity is much weaker than that of VP3. VP3 is a $13 \mathrm{kDa}$ protein, also named

\section{C) Biomed Central}


apoptin, which induces apoptosis in infected chicken cells and human tumor cell lines $[2,18,19]$. During virus infection, VP2 and VP3 are detected very early, namely $12 \mathrm{~h}$ post infection, while VP1 is detected only after 30 $h$ post infection [16]. Some additional proteins have been reported to be translated after further splicing of the mRNA, but their biological functions have not been elucidated [20].

As mention above, VP2 is a DSP enzyme [17]. The key catalytic residues of active site have been identified to be serine, threonine, and tyrosine. The cysteine residues, respectively, are located at positions 95 and 97 in the catalytic motif of VP2. Furthermore, mutation of these residues to serine results in reduced virus replication efficiency in the cell [17]. This effect indicates that the phosphatase activity of VP2 is required for virus replication. It has been reported that co-expression of VP1 and VP2 allows neutralizing antibodies to be raised $[21,22]$ and it has been suggested that VP2 is a scaffold protein [23] that corrects the conformation of VP1. Therefore, it is expected that VP2 is a multifunctional protein with roles in virus infection, assembly and replication. VP2 possess a putative NLS and the protein is known to accumulate to a large extent in the nucleus of infected chicken cells $[16,24]$. A recent study has shown that VP2, when fused to GFP, shows nuclear localization and this result indicates that the NLS of VP2 is also functional in plants [25].

Until now, the specific mechanism for the cellular localization of VP2 is not well understood. In this study, we first use bioinformatics to analysis the amino acid sequence of various different isolates of VP2, and were able to predict and examine for the presence of putative NLS and NES motifs, which have never been characterized previously. We generated GFP fused to various versions of VP2 created by truncation, site directed mutagenesis, and multiple site directed mutagenesis in order to confirm the locations of these putative NLS and NES sequences. Leptomycin B (LMB) can be used to identify the presence of a NES motif in a protein because it inhibits the CRM1 pathway and such a result has been found for VP3 of CAV. Using LMB, our results suggest that VP2 does not contain a functional NES and also that VP2 is CRM1 independent. Additionally, using a co-immunoprecipitation assay, we also found that VP2 associates with MCM3 and that this interaction does not require DSP activity.

\section{Results}

\section{Localization of VP2 in mammalian cells}

In previous studies, it has been shown by indirect immunoperoxidase staining that VP2 is localized within the nuclei of MDCC-MSB 1 cells infected with CAV [16]. In 2007, Lacorte et al. reported that transient expression of
GFP-VP2 could be observed throughout the nucleoplasm in plant cells [25]. In the present study, we constructed expression plasmids of VP2-GFP in order to identify the nuclear localizing characteristics in cells. After transient transfection, the bright fluorescence of VP2-GFP was observed mostly in the nucleus of both HeLa and $\mathrm{CHO}$ cells. In contrast, when GFP alone was introduced, not only the nucleus but also the cytoplasm was fluorescent. This indicates that a functional NLS was present in VP2 as suggested by previously (Figure 1A and 1B).

\section{Using bioinformatics to predict the NLS and NES containing regions}

In order to identify if there are any NLS or NES motifs in VP2, we first compared the amino acid sequence of VP2 (Taiwan CIA-89) with a range of other CAV isolates to explore the protein's sequence divergence. The various VP2 sequences of the different CAV isolates were obtained from the UniProtKB database (http:// www.uniprot.org/). Based on the sequence alignment, the VP2 proteins of these CAV isolates are highly conserved compared to strain CIA- 89 from Taiwan. Therefore, the full length of amino acid sequence of VP2 (Taiwan CIA-89) was used and examined in order to predict NLS sequences using the WoLF PSORT and NLStradamus programs (Figures 2 and 3A). A bipartite NLS motif (named BiNLS1) was predicted by the WoLF PSORT program, with the putative motif position spanned amino acid residues from 136 to 153 (under line). However, a monopartite NLS motif (named NLS2) was also predicted by NLStradamus at a prediction cutoff value at 0.5 and this motif was located from amino acid residues from 133 to 138 (bold). Based on the results of bioinformatics analysis, VP2 was predicted to containing two possible NLS motifs. In addition, the NES motif prediction was performed by NetNES 1.1 Server (Figures 2 and 3A). A putative NES from amino acid residues 120 to 128 (under line, bold, shadow and Italic) was pinpointed. However, the expected value for NES prediction was lower than threshold expected value of this program, which suggests that there is a low probability of a NES motif existing within VP2. Based on these results, further investigations were needed to elucidate whether or not a NES is functional in VP2.

\section{Identification of BiNLS1 function in VP2}

As mentioned above, two putative NLS motifs, a bipartite BiNLS1 and a monopartite NLS2 were predicted to be present in VP2. To determine the exactly site of the NLS motif in VP2, we constructed a full length clone and six deletion clones of VP2 fused with GFP at the C-terminus (Figure 3A). The subcellular locations of these expressed constructs in the transfected cells based on the GFP distribution pattern at $48 \mathrm{~h}$ post-transfection were examined. 


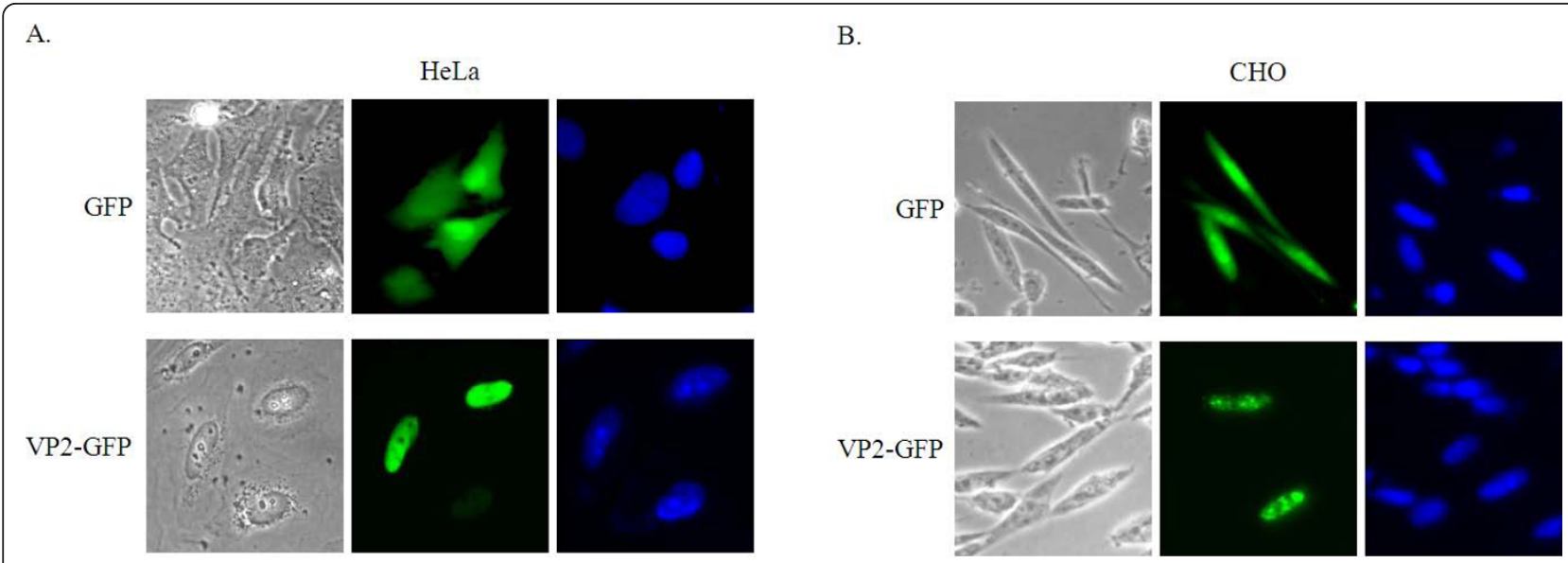

Figure 1 VP2 protein is localized to the nucleus of the mammalian cells. HeLa (A) and CHO (B) cells both were transfected with GFP and VP2-GFP expressing plasmids (green). At $48 \mathrm{~h}$ post-transfection, the cells were fixed and stained with DAPI (blue). The distribution of GFP and VP2-GFP in the cells were followed by microscopy (phase) and fluorescence microscopy

The truncated C-terminal deletion of VP2-GFP fusion proteins were VP2 115dC, VP2 132dC, and VP2 145dC. The N-terminal deletion mutants were VP2 111dN, VP2 $141 \mathrm{dN}$, and VP2 160dN. In Figure 3B, two of the C-terminal deletions, VP2 115dC, and VP2 132dC showed fluorescence distributed in both the nucleus and cytoplasm of
HeLa cells (Figure 3B), whereas the bright fluorescence of these two truncations was predominantly distributed in the cytoplasm of $\mathrm{CHO}$ cells (Figure 3B). Fluorescence due to VP2 $145 \mathrm{dC}$ was localized in the nucleus of both HeLa and $\mathrm{CHO}$ cells (Figure 3B). Furthermore, VP2 111dN was also localized to the nucleus of HeLa and $\mathrm{CHO}$ cells

\begin{tabular}{|c|c|c|}
\hline & & $\nabla \sqrt{100}$ \\
\hline Taiwan CIA-89 & $(51)$ & VQATNKFTAVGNPSLQRDPDWYRWNYNHS I AVWLRE CSRSHAKI CNCGQF \\
\hline Australia/CAU269-7/2000 & $(51)$ & VQATNKFTAVGNPSLQRDPDWYRWNYSHS I AVWLRECSRSHAKI CNCGQF \\
\hline \multirow[t]{5}{*}{ Germany } & $(51)$ & VQATNKFTAVGNPSLQRDPDWYRWNYNHS I AVWLRECSRSHAKI CNCGQF \\
\hline & $(51)$ & VQATNKFTAVGNPSLQRDPDWYRWNYNHS I AVWLRECSRSHAKI CNCGQF \\
\hline & $(51)$ & VQATNKFTAVGNPSLQRDPDWYRWNYNHS I AVWLRECSRSHAKI CNCGQF \\
\hline & $(51)$ & VQATNKFTAVGNPSLQRDPDWYRWNYNHS I AVWLRECSRSHAKI CNCGQF \\
\hline & & 101 \\
\hline Taiwan CIA-89 & $(101)$ & RKHWFQE CAGLEDRSTQASLEEAILRPLRVQGKRAKRKLDYHYSQPTPNR \\
\hline Australia/CAU269-7/2000 & $(101)$ & RKHWFQE CAGLEDRSTQASLEEAILRPLRVQGKRAKRKLDYHYSQPTPNR \\
\hline \multirow[t]{5}{*}{ Germany } & $(101)$ & RKHW F QE CAGLEDRSTQASLEEAILRPLRVQGKRAKRKLDYHYSQPTPNR \\
\hline & $(101)$ & RKHWFQE CAGLEDRSTQA SLEEAILRPLRVQGKRAKRKLDYHYSQPTPNR \\
\hline & $(101)$ & RKHWFQE CAGLEDRSTQASLEEAILRPLRVQGKRAKRKLDYHYSQPTPNR \\
\hline & $(101)$ & RKHWFQE CAGLEDRSTQA SLEEAILRPLRVQGKRAKRKLDYHYSQPTPNR \\
\hline & & 151 \\
\hline Taiwan CIA-89 & $(151)$ & KKVYKTVRWQDELADREADFTPSEEDGGT TSSDFDEDINFDIGGDSG IVD \\
\hline Australia/CAU269-7/2000 & $(151)$ & KKVYKTVRWQDELADREADFTPSEEDGGT TSSDFDEDINFDI GGDSG I VD \\
\hline \multirow{4}{*}{ Germany } & $(151)$ & KKVYKTVRWQDELADREADFTPSEEDGGTTSSDFDEDINFDIGGDSG IVD \\
\hline & $(151)$ & KKVYKTVRWKDELADREADFTPSEEDGGT TSSDFDEDINFDI GGDSG IVD \\
\hline & $(151)$ & KKVYKTVRWQDELADREADFTPSEEDGGTTSSDFDGDINFDIGGDSG IVD \\
\hline & $(151)$ & KKVYKTVRWQDELADREADFTPSEEDGGT TSSDFDEDINFD I GGDSG I VD \\
\hline \multicolumn{3}{|c|}{$\begin{array}{l}\text { Figure } 2 \text { Analysis and predication of NLS and NES motifs present in the VP2 amino acid sequence. The various VP2 amino acic } \\
\text { sequences ( } 51 \text { to 200) from different CAV isolates were aligned as described in the Materials and Methods. The putative NLS motifs (BiNLS1: } \\
\text { under line and NLS2: bold words) and NES motifs (under line, bold, shadow and Italic) are shown. The cysteine residues at positions } 95 \text { and } 97 \\
\text { in the catalytic motif of VP2 are also indicated by an arrow and an arrow head, respectively }\end{array}$} \\
\hline
\end{tabular}




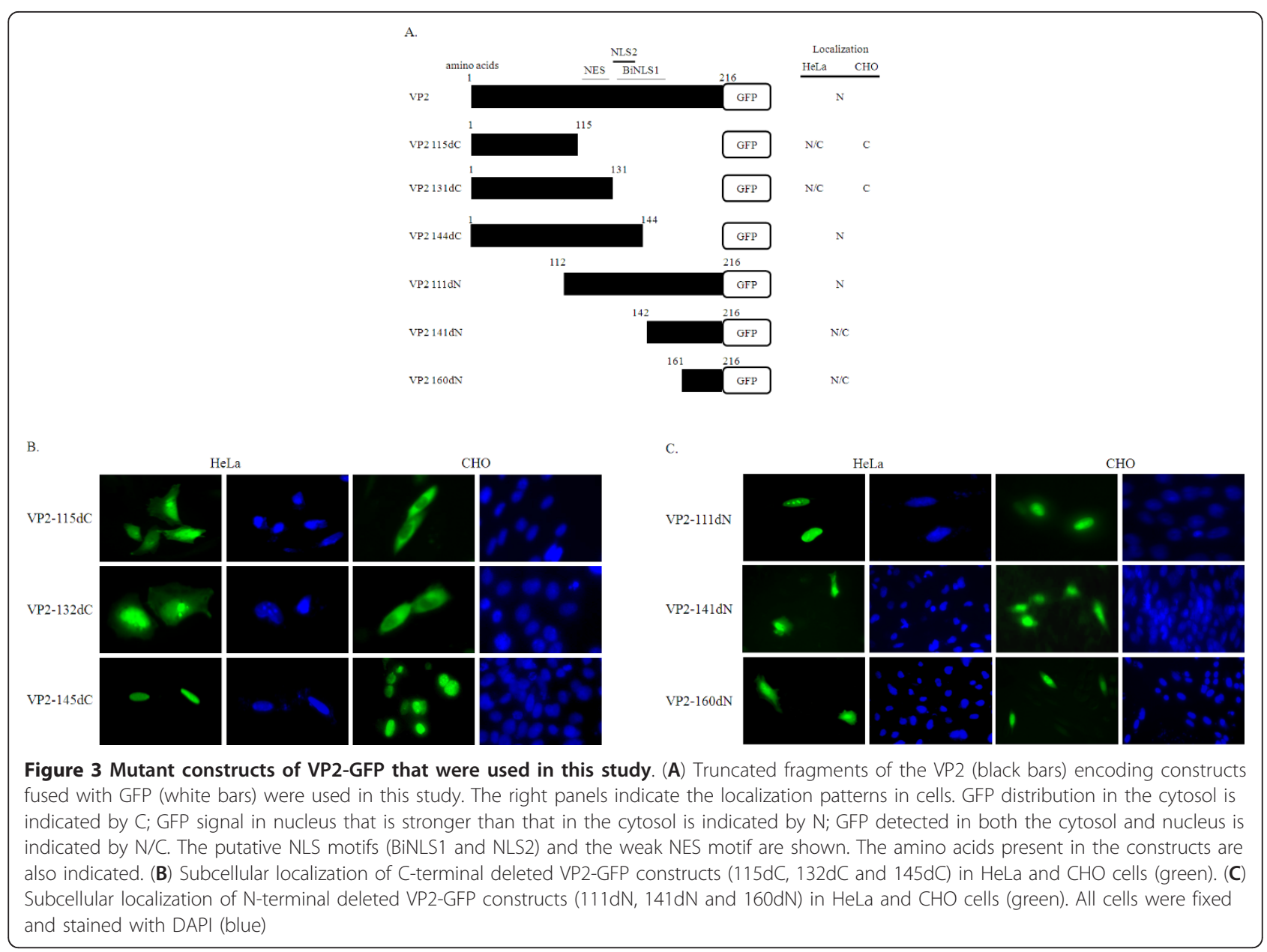

(Figure 3C). In contrast, fluorescence by VP2 $141 \mathrm{dN}$ and VP2 $160 \mathrm{dN}$ was found to have a very similar pattern to that of GFP in both the nucleus and cytoplasm of HeLa and $\mathrm{CHO}$ cells (Figure $3 \mathrm{C}$ ).

On comparing the fluorescence distribution patterns of VP2 111dN and VP2 141dN in mammalian cells, we found that the C-terminus part of BiNLS1 from VP2 did not seem to contain a nuclear import function. To support this finding, we used site directed mutagenesis to create a series of BiNLS1 mutants, namely VP2 150152A (R150A, K151A and K152A), VP2 136-138A (K136A, R137A, and K138A) and VP2 136-138A/150$152 \mathrm{~A}$, as shown in Table 1 . All of these mutants were able to express and were localized to the nucleus of HeLa and $\mathrm{CHO}$ cells (Figure 4A). The results indicated that NLS motif did not include the whole putative region of BiNLS1 as predicted by bioinformatics.

The nuclear localization signal is within the NLS2 motif of VP2

Next, we focused on NLS2 motif in order to characterize the NLS motif within VP2. The VP2 132dC mutant produced fluorescence that was distributed across both the nucleus and cytoplasm of HeLa cells and in the cytoplasm of $\mathrm{CHO}$ cells. In contrast, the fluorescence produced by VP2 $145 \mathrm{dC}$ was localized to the nucleus of HeLa and CHO cells. These results indicate that NLS motif of VP2 would seem to span amino acid residues 132 to 144 . This region contains the NLS2 motif, which covers amino acid residues 133 to 138 . We changed the positive charge of amino acids from 136 to 138 residues to alanine and measured the distribution of GFP. We found that the nuclear localization of GFP fluorescence of the VP2 136-138A mutants within this region was not abolished. However, our results indicated the presence of critical amino acid residues within the NLS motif of VP2 that play an essential role in nuclear transport. Specifically, alanine substitution mutagenesis at positions K133A and R134A of VP2 and of the VP2 136-138A clone was carried out to further characterize the nuclear localization motif and the results were summarized in Table 1. These results were shown that nuclear transportation of VP2 was obviously disrupted in three alanine substitution constructs, namely VP2 
Table 1 Intracellular localization of VP2 and various mutants of the NLS motif

\begin{tabular}{|c|c|c|}
\hline Name of mutants & Localization $^{\mathrm{a}}$ & NLS motifs of amino acids sequence ${ }^{b, c}$ \\
\hline VP2-GFP & $\mathrm{N}$ & $K^{133} R^{134} A^{135} K^{136} R^{137} K^{138} L^{139} D^{140} Y^{141} H^{142} Y^{143} S^{144} Q^{145} P^{146} T^{147} P^{148} N^{149} R^{150} K^{151} K^{152}$ \\
\hline VP2 150-152A & $\mathrm{N}$ & $K^{133} R^{134} A^{135} K^{136} R^{137} K^{138} L^{139} D^{140} Y^{141} H^{142} Y^{143} S^{144} Q^{145} P^{146} T^{147} P^{148} N^{149} A^{150} A^{151} A^{152}$ \\
\hline VP2 136-138A & $\mathrm{N}$ & $K^{133} R^{134} A^{135} A^{136} A^{137} A^{138} L^{139} D^{140} Y^{141} H^{142} Y^{143} S^{144} Q^{145} P^{146} T^{147} P^{148} N^{149} R^{150} K^{151} K^{152}$ \\
\hline VP2 136-138A/150-152A & $\mathrm{N}$ & $K^{133} R^{134} A^{135} A^{136} A^{137} A^{138} L^{139} D^{140} Y^{141} H^{142} Y^{143} S^{144} Q^{145} P^{146} T^{147} P^{148} N^{149} A^{150} A^{151} A^{152}$ \\
\hline VP2 136-138A/133A & C & $A^{133} R^{134} A^{135} A^{136} A^{137} A^{138}$ \\
\hline VP2 136-138A/134A & C & $K^{133} A^{134} A^{135} A^{136} A^{137} A^{138}$ \\
\hline VP2 136-138A/133A/134A & C & $A^{133} A^{134} A^{135} A^{136} A^{137} A^{138}$ \\
\hline VP2 133A & $\mathrm{N}$ & $A^{133} R^{134} A^{135} K^{136} R^{137} K^{138}$ \\
\hline VP2 134A & $\mathrm{N}$ & $K^{133} A^{134} A^{135} K^{136} R^{137} K^{138}$ \\
\hline VP2 133A/134A & $\mathrm{N}$ & $A^{133} A^{134} A^{135} K^{136} R^{137} K^{138}$ \\
\hline
\end{tabular}

136-138A/133A, VP2 136-138A/134A, and VP2 136138A/133A/134A (Figure 4B) but not in VP2 133A, VP2 134A and VP2 133A/134A (Figure 4C). The GFP signal distributions of VP2-GFP and its mutants in $\mathrm{CHO}$ cells are shown in Figures $1 \mathrm{~B}$ and $4 \mathrm{~B}$ and these were analyzed quantitatively by using Alpha View ${ }^{\circledR}$ Software (Figure 5). VP2-GFP was $94 \%$ of the time in nucleus and $6 \%$ in cytosol. The nuclear presence of the VP2 136-138A/ 133A, VP2 136-138A/134A, and VP2 136-138A/133A/ $134 \mathrm{~A}$ mutant was reduced to $13 \%, 12 \%$ and $11 \%$, respectively, with most of GFP signal accumulated in the cytoplasm. These findings demonstrate that NLS2 is a functional NLS motif in VP2 and that amino acid residues K133 and R134 are also important to this functionality (Figures 4B and 5).

\section{VP2 is a CRM1 independent protein}

Typical NES motifs have been found in the various viral and cellular proteins and are involved in transportation between the nucleus and the cytoplasm [26]. LMB has been shown to interfere with the CRM1-NES interaction and can be used to verify the functionality of any NES motif. A weak putative NES motif spanning amino acid residues from 120 to 128 of VP2 was predicted earlier (Figure 2). However, the prediction score was lower than the program threshold. The weak putative NES motif was investigated using the truncated mutants shown in Figure 3A together with LMB (+) $(20 \mathrm{ng} / \mathrm{ml})$ and LMB (-) (PBS) buffers, which were added to cells at $48 \mathrm{~h}$ post-transfection and incubated for $1 \mathrm{~h}[27,28]$. VP3-GFP was used as a positive control because it has been reported to be a CRM1 dependent protein and contains a NES motif $[29,30]$. The distribution of fluorescence was then observed (see Additional file 1) and the results demonstrated that, while LMB was able to affect the nuclear export of VP3, it had no effect on VP2. Therefore it would seem that VP2 nuclear export is a CRM1 independent process and that there is no functional NES motif present in VP2.

\section{VP2 binds to chromatin}

As previously reported and confirmed by this study, VP2-GFP predominantly is localized in nucleus (Figure $1)$. In order to further investigate this localization, $\mathrm{CHO}$ cells were transfected with VP2-GFP plasmid and the resulting cell extract separated into soluble and chromatin fractions. As is shown in Figure 6A, VP2-GFP could be detected in both the soluble and chromatin fractions. The chromatin fraction was further digested with micrococcal nuclease (MNase). Like MCM3, VP2-GFP was only partial dissociated from the chromatin fraction after treatment with MNase, which suggested that VP2 is a chromatin-bound protein and MNase-resistant. The chromatin fraction was also treated with different concentration of $\mathrm{NaCl}$. The result is shown in Figure 6A, whereby VP2-GFP was found to be slightly dissociation at $0.3 \mathrm{M} \mathrm{NaCl}$ and $0.5 \mathrm{M} \mathrm{NaCl}$, but a significant amount of remaining protein remained bound to the chromatin. When tested under similar conditions, the lamin B receptor, a nuclear matrix protein, was not extracted by $0.3 \mathrm{M} \mathrm{NaCl}$ and $0.5 \mathrm{M} \mathrm{NaCl}$. Therefore, these results strongly suggest that VP2, like MCM3, is a chromatin binding protein, but was not a nuclear matrix protein.

\section{The DSP activity of VP2 is not required for protein- protein interaction with MCM3}

In a previous study, the DSP activity of VP2 was shown to be needed for virus replication in the cell [28]. In order to address what proteins in the various DNA replication complexes interacts with VP2 in cells, an independent assay involving immunoprecipitation using anti-Flag M2 beads against Flag-VP2-GFP was employed. In the control experiments, GFP expressing 


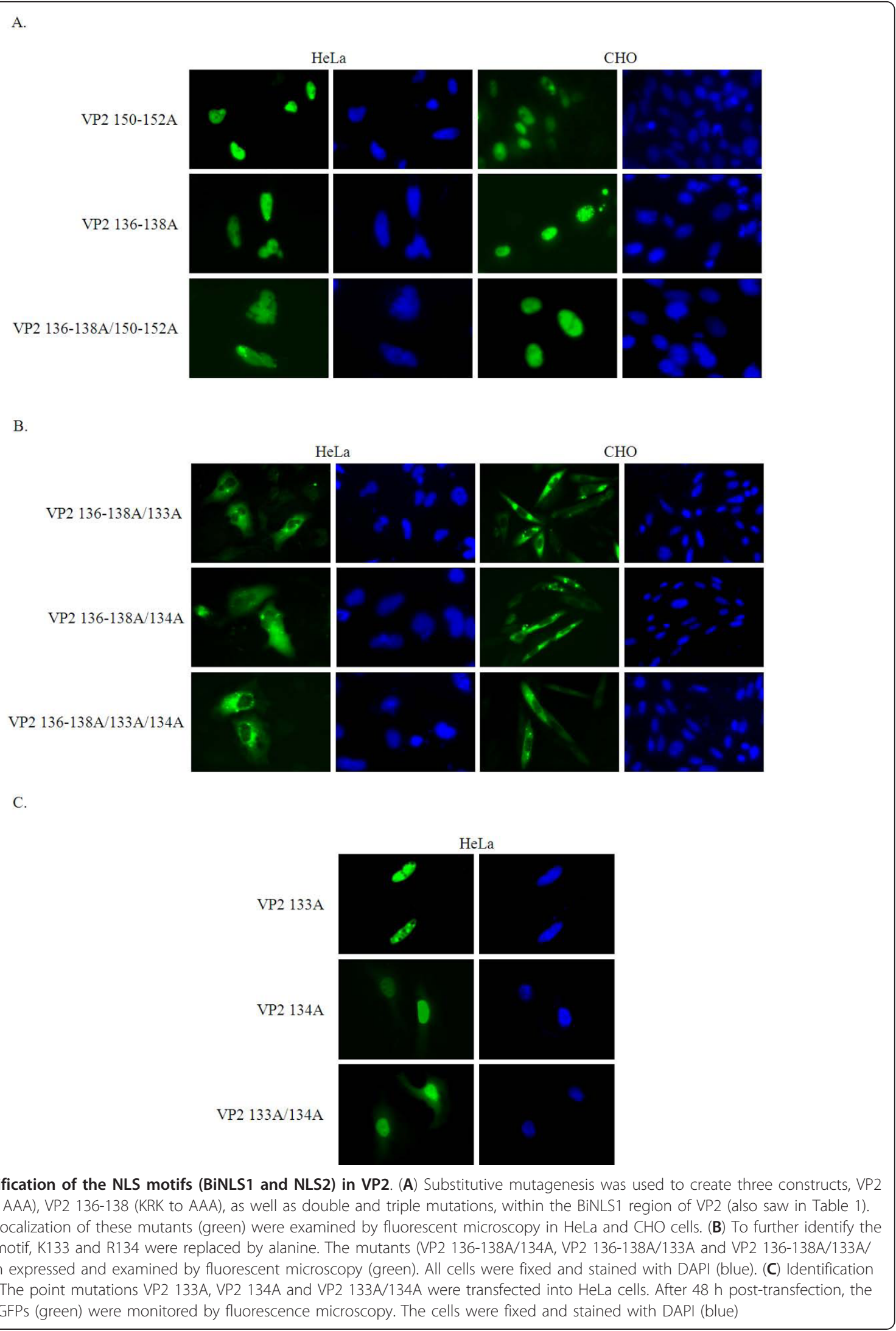




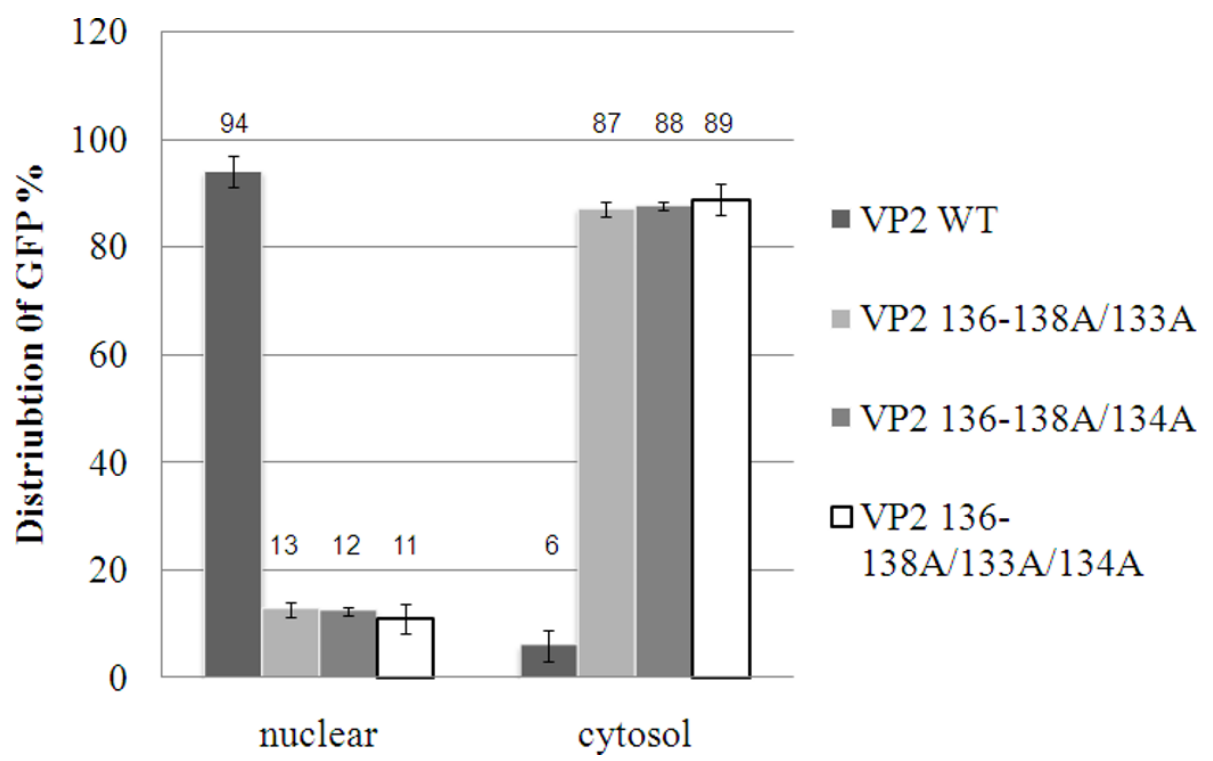

Figure 5 The distribution of VP2-GFP and mutants in cytoplasm and nucleus of cells. VP2-GFP and various mutants (VP2 136-138A/134A, VP2 136-138A/133A and VP2 136-138A/133A/134A) were transfected into CHO cells. After $48 \mathrm{~h}$ transfection, all cells were fixed and stained with DAPI. The distribution of GFP was monitored by fluorescence microscopy. In VP2-GFP and mutants, twenty cells were measured the distribution of GFP signals from cytoplasm and nucleus using Alpha View ${ }^{\mathbb{B}}$ Software (Alpha Innotech Corporation)

plasmids were transfected into cells and it was found that no none-specific proteins were brought down by the Flag M2 beads using Western blot detection (Figure $6 \mathrm{~B}$, lane Control). On the other hand, Flag-VP2-GFP was co-immunoprecipited with MCM3 protein by the Flag M2 beads (Figure 6B, lane WT) when compared to the protein band in the Flag-VP2-GFP nuclear extract (Figure 6B, lane $\mathrm{N}$ ). In contrast, other components of DNA replication complexes such as $\mathrm{CDC7}, \mathrm{PCNA}$ and condensin SMC2, as well as the core histones such as $\mathrm{H} 2 \mathrm{~B}$, were not co-immunoprecipited with VP2 using $\mathrm{CHO}$ cell extracts (see Additional file 2). The above results confirmed that VP2 associates with MCM3 when it binds to chromatin.

To identify whether the dual phosphatase activity of VP2 was needed for association with MCM3, we performed a site directed mutagenesis assay targeting position $\mathrm{C} 95$ and C97 of VP2 in order to create the single mutants C95S and $\mathrm{C} 97 \mathrm{~S}$ and the double mutant C95S/C97S, which have either partial or complete disruption of the DSP activity associated with VP2 [17]. Co-immunoprecipitation assays were carried out and, the results are shown in Figure 6C, MCM3 was co-immunoprecipitated by the three mutants of VP2 protein in a similar manner to the wild type protein, which shows that VP2 binding to MCM3 does not require dual-phosphatase activity (Figure 6C).

\section{Discussion}

CAV is an important avian pathogen worldwide and causes major economic damage throughout the poultry industry. Three major proteins are encoded by this virus, namely the capsid protein VP1, the nonstructural protein VP2, and the apoptin VP3. However, up to the present, the exactly functions of these three proteins in cell are still poorly understood. In previous studies, the expression of VP2 was found to be nuclear in plant cells [25]. This result is similar to that of the present study, where, VP2 was found to accumulate in the nucleus of both HeLa and CHO cells. Our results, both by bioinformatics and site-directed mutagenesis, support the hypothesis that VP2 contain a NLS and named as NSL2. NLS2 was shown to be a functional NLS that allowed transfer of VP2 into nucleus. This is similar to VP3, which has two functional NLS motifs that are also used for nuclear localization. The single VP2 NLS motif spans amino acid residues 133 to 138 . Within this motif, we also found that two amino acids with positive charges, 133R and $134 \mathrm{~K}$ were important to allowing VP2 protein to shuttle from the cytoplasm to the nucleus. To the best of our knowledge, this is the first report identifying a NLS motif in the VP2 of CAV.

Subsequent sequence analysis showed that VP2 might contain a putative CRM1-mediated NES motif that stretched from amino acid 120 to amino acid 128, however the scores for this motif was below the program threshold. In order to confirm that a NES motif really existed in VP2, we used fusion protein constructs to investigate nuclear transportation in the presence of LMB assay (Figures $3 \mathrm{~A}$ and see Additional file 1). A control protein, namely VP3, also named apoptin, which 
A.

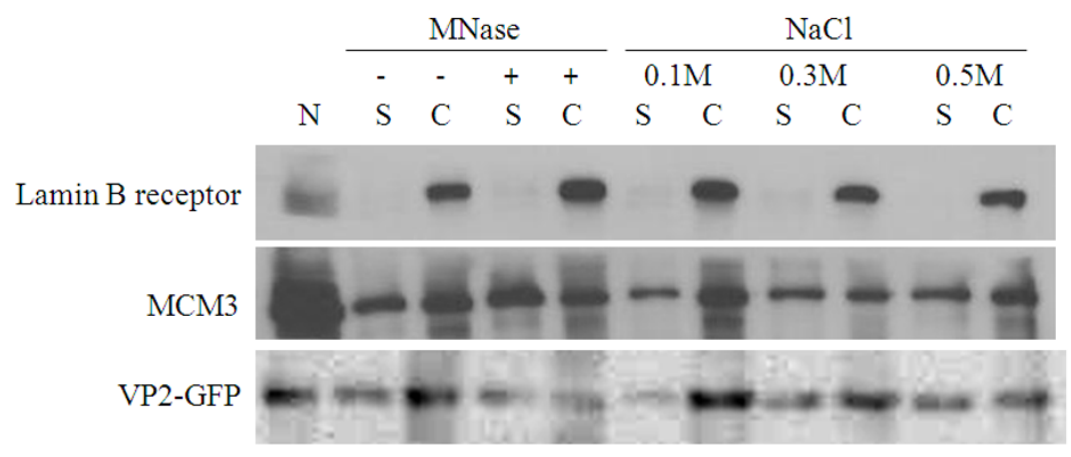

B.

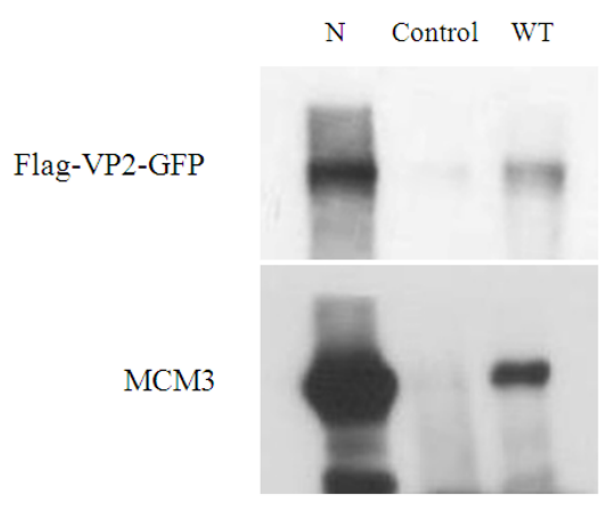

C.

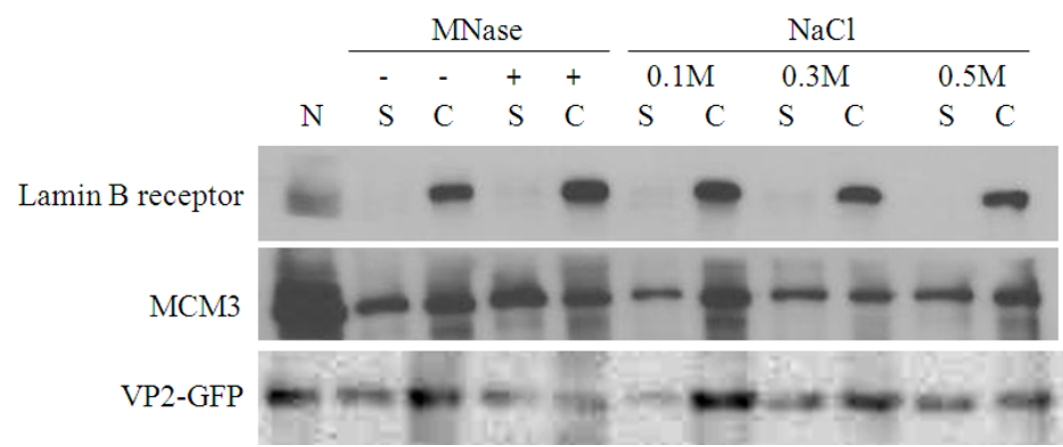

Figure 6 VP2 binds to chromatin and interacts with MCM3 but this does not require dual phosphatase activity. (A) The soluble (S) and chromatin (C) fractions were prepared by using CSK buffer containing $0.5 \%$ Triton X-100 in CHO cells of transfected with VP2-GFP plasmid. The fractions were treated with $\mathrm{U}(-)$ and $150 \cup(+)$ of MNase or the indicated concentrations of $\mathrm{NaCl}$. Nuclear extracts (N) containing VP2-GFP were as positive control and all fractions were subjected to immunoblotting against with lamin B receptor, MCM3, and VP2 antibodies. (B) At 48 h post-transfection, the GFP (as Control) and Flag-VP2-GFP (as wild-type; WT) were found in the CHO cells. Next the lysates were immunoprecipitated using Flag M2 beads and immunoblotted against VP2 and MCM3 antibodies. (C) The WT and mutants (C95S, C97S, and C95S/C97S) of the Flag-VP2-GFP in CHO cells were also examined at $48 \mathrm{~h}$ post-transfection. The cell lysates were immunoprecipitated using Flag M2 beads and immunoblotted against with VP2 and MCM3 antibodies. The arrow head was indicated a none-specific band. The nuclear extracts containing VP2-GFP were designated as N 
contains a classical leucine-rich NES motif and has been previously described as sensitive to LMB treatment was included in the study $[29,30]$. Our results confirmed that the cytoplasm and nuclear distribution of the various truncated VP2 mutants was not obviously changed in pattern after treatment with LMB. This supports two possibilities; firstly, that the nuclear export pathway (using NES motif) of VP2 might not exist and/or, secondly, that a distinct and CRM1 dependent pathway was adopted by VP2 for nuclear export.

VP2 has been shown to contain dual phosphatase activity and this activity is required for CAV replication $[17,31]$. However, in this context, very little is known about the role this protein plays in the regulation of viral DNA replication. When cells are infected with CAV, VP2 is expressed and accumulates, reaching a detectable level at $12 \mathrm{~h}$ post-infection $[8,16]$. In contrast, VP1 has been found to accumulate to a higher level at $30 \mathrm{~h}$ post-infection. Amino acids sequence analysis of CAV VP1 was revealed that the N-terminal region show similarity to protamines [11], this supports the hypothesis that VP1 has DNA-binding functionality [32]. The $\mathrm{N}$ and C-terminal domains of VP3 separately bind to DNA and indicating the presence of multiple independent binding sites [33]. However, VP2 protein has no obvious DNA binding characteristics. In this study, VP2 of CAV is shown to be localized to the nucleus in two cell lines (Figure 1) and also to bind to chromatin (Figure 6). The latter finding was confirmed by examining the nuclease-resistant fraction and by treating with high salt. VP2 is similar to MCM3, a component of various MCM2-7 complexes, and other nuclease-resistant chromatin bound proteins such as CDC6 [18], minichromosome maintenance proteins (MCMs) [34], and PCNA [35], all of which have chromatin-bound characteristics. This suggests that the early expression of VP2 might involve DNA replication with VP2 interacting with the prereplication complex (Figure 6B). The association of VP2 with MCM3 (and perhaps other members of a MCM2-7 complex) may facilitate the DNA replication of CAV. However, it is still unknown whether VP2 is able to bind to DNA directly and this will need further investigation. Moreover, VP2's association with MCM3 does not require dual phosphatase activity (Figure 6C) and therefore, the relevance of VP2's DSP activity to the live cycle of CAV also needs further investigation.

Rep, a viral protein with replicase activity involved in regulating rolling-circle replication (RCR), has been found in most circovirus members of the family Circoviridae [36]. CAV seems to lack a Rep protein. However, some studies have proposed that the C-terminus of CAV VP1 contains a highly conserved RCR motif and that this may play a role in regulating the RCR reaction [32]. This RCR-regulating function of VP1 seems to be very similar to that of the Rep protein. Therefore, VP1 may interact with VP2 to form a nucleoprotein complex and, in addition, the genomic DNA of the virus might also be coupled with VP1 and VP2 in order to regulate the RCR reaction during the early stages of infection. However, these hypotheses need to be investigated in terms of how the MCMs interact with the VP2 of CAV.

\section{Conclusions}

We have demonstrated that the VP2 of CAV contains a functional NLS motif that spans amino acids 133 to 138 of the protein. In addition to having a NLS, VP2 is also a chromatin binding protein similar to members of the MCM2-7 complex. Moreover, VP2 associates with MCM3 in cells based on co-immunoprecipitation analysis. Taken together, these findings suggest that VP2 may be part of a DNA pre-replication complex.

\section{Methods}

\section{Antibodies}

All primary anti-human and mouse antibodies used for immunoblotting were purchased from commercial companies. Rabbit polyclonal anti-human and mouse antibodies were obtained as follows: $\mathrm{SMC} 2, \mathrm{CDC7}$, and $\mathrm{H} 2 \mathrm{~B}$ from Santa Cruz Biotechology, USA; MCM3 from Bethyl Laboratories, USA; PCNA from Epitomics, USA and rabbit monoclonal anti-human and mouse lamin $B$ receptor antibody from Abcam, USA. The anti-VP2 of $\mathrm{CAV}$ polyclonal antibody was prepared by immunizing rabbits using VP2 of CAV expressed in E. coli [37]. The secondary antibodies for Western blotting were as follows: the goat anti-mouse IgG and goat anti-rabbit IgG conjugated HRP, which were both obtained from Santa Cruz Biotechology, USA.

\section{Construction of plasmids}

Various plasmids, such as pcDNA3.1 VP2-GFP, pcDNA3.1 Flag-VP2-GFP and pcAcGFP1-N1 VP3-GFP, were constructed as described below. The full-length of VP2, Flag-VP2 and VP3 were amplified by PCR using high fidelity Platinum Taq DNA polymerase (Invitrogen, USA) from pGEX-6P-1-VP2 [38] and pGEX-6P-1-VP3 (data not shown) using the primers: forward VP2 1-18 EcoRI 5' TGGAATTCATGCACGGGAACGGCGGA3' or VP2 Flag EcoRI 5' AGGATCCATGGATTACAAGGATGACGACGATAAGGAATTCATGCACGG-

GAACGGCGGACA 3' and reverse VP2 657 XhoI 5' TCCTCGAGCACTATACGTACCGG 3' for VP2 and Flag-VP2 in addition to forward VP3 NheI 5' AGCTAGCATGAACGCTCTCCAAGAAG 3' and reverse VP3 Xhol 5' TCCTCGAGCAGTCTTATACACCTTCT 3' for VP3. The PCR products were then ligated into the yT\&A vector (Yeastern Biotech, Taiwan). The EcoRI/ XhoI fragments containing VP2 and Flag-VP2 or NheI/ 
XhoI fragments containing VP3 were released from the yT\&A vector and ligated into pcDNA3.1-GFP, which was a kind gift from Prof. Min-Ying Wang (the Graduate Institute of Biotechnology, National Chung Hsing University, Taichung, Taiwan) and pAcGFP1-N1 (Clontech, USA). The truncated and point mutations of the VP2-GFP constructs were generated in a similar manner to that described above and the primers are summarized in Table 2. Single site directed and multiple site directed mutagenesis were carried out by PCR using PfuUltra ${ }^{\mathrm{TM}}$ High-Fidelity DNA Polymerase (Stratagene, USA) in addition to using pcDNA3.1 VP2-GFP and pcDNA3.1 Flag-VP2-GFP as the template with the primers in Table 2.

\section{Amino acid sequence analysis and predication}

The amino acid sequences of VP2 from different isolates were identified by searching the UniProtKB database (available at http://www.uniprot.org/). The isolates analyzed were Taiwan CIA-89 (in the present paper), Australia/CAU269-7/2000 (accession number: Q9IZU7), Germany Cuxhaven-1(accession number: P69484), Japan 82-2 (accession number: P54093), USA 26p4 (accession number: P54092), and USA CIA-1(accession number: P69485). The amino acid sequences were then aligned and analyzed using program of Biology Workbench 3.2 (San Diego Supercomputer Center; SDSC). The putative NES motif was predicted by the NetNES 1.1 Server [39] while the NLS was predicted by WoLF PSORT [40] and NLStradamus [41].

\section{Cell culture, transfection and staining}

Chinese hamster ovary (CHO) cells were grown in GIBCO $^{\circledR}$ Dulbecco's Modified Eagle Medium: Nutrient Mixture F-12 (DMEM/F-12) medium (Invitrogen, USA) supplemented with $10 \%(\mathrm{v} / \mathrm{v})$ fetal bovine serum (FBS) (GIBCO/Invitrogen, USA), 100 units/ml penicillin, and $100 \mu \mathrm{g} / \mathrm{ml}$ streptomycin. HeLa cells were grown in

Table 2 The primers used to create the various truncated, single and multiple mutants by PCR in this study

\begin{tabular}{|c|c|c|c|}
\hline Primer name & Type & Length & Sequence $\left(5^{\prime}-3^{\prime}\right)$ \\
\hline VP2 $111 \mathrm{~N}$ del EcoRI & Forward & 26-mer & TGGAATTCATGGAGGACCGATCAACC \\
\hline VP2 $141 \mathrm{~N}$ del EcoRI & Forward & 26-mer & AGGAATTCATGCACTACTCCCAGCCG \\
\hline VP2 $160 \mathrm{~N}$ del EcoRI & Forward & 26-mer & AGGAATTCATGGACGAGCTCGCAGAC \\
\hline VP2 115 C del Xhol & Reverse & 24-mer & TCCTCGAGTGATCGGTCCTCAAGT \\
\hline VP2 132 C del Xhol & Reverse & 23-mer & TCCTCGAGACCCTGTACTCGGAG \\
\hline VP2 145 C del Xhol & Reverse & 26-mer & TCCTCGAGCTGGGAGTAGTGGTAATC \\
\hline VP2 136-138A & Forward & 27-mer & AAACGAGCTGCTGCTGCTCTTGATTAC \\
\hline VP2 136-138A & Reverse & 27-mer & GTAATCAAGAGCAGCAGCAGCTCGTTT \\
\hline VP2 150-152A & Forward & 39-mer & ACCCCGAACGCAGCAGCAGTGTATAAGACTGTAAGATGG \\
\hline VP2 150-152A & Reverse & 39-mer & CCATCTTACAGTCTTATACACTGCTGCTGCGTTCGGGGT \\
\hline VP2 136-138A/134/A & Forward & 27-mer & GTACAGGGTAAAGCTGCTGCTGCTGCT \\
\hline VP2 136-138A/134/A & Reverse & 27-mer & AGCAGCAGCAGCAGCTTTACCCTGTAC \\
\hline VP2 136-138A/133/A & Forward & 27-mer & GTACAGGGTGCTCGAGCTGCTGCTGCT \\
\hline VP2 136-138A/133/A & Reverse & 27-mer & AGCAGCAGCAGCTCGAGCACCCTGTAC \\
\hline VP2136-138A/133A/134/A & Forward & 27-mer & GTACAGGGTGCTGCTGCTGCTGCTGCT \\
\hline VP2 136-138A/133A/134/A & Reverse & 27-mer & AGCAGCAGCAGCAGCAGCACCCTGTAC \\
\hline VP2 133A & Forward & 28-mer & GTACAGGGTGCTCGAGCTAAAAGAAAGC \\
\hline VP2 133A & Reverse & 28-mer & GCTITCTITIAGCTCGAGCACCCTGTAC \\
\hline VP2 134A & Forward & 28-mer & GTACAGGGTAAAGCTGCTAAAAGAAAGC \\
\hline VP2 134A & Reverse & 28-mer & GCTITCTITTAGCAGCTITACCCTGTAC \\
\hline VP2 133A/134A & Forward & 28-mer & GTACAGGGTGCTGCTGCTAAAAGAAAGC \\
\hline VP2 133A/134A & Reverse & 28-mer & GCTTTCTTITAGCAGCAGCACCCTGTAC \\
\hline VP2 C95S & Forward & 20-mer & CGCTAAGATCAGCAACTGCG \\
\hline VP2 C95S & Reverse & 22-mer & CGCAGTTGCTGATCTTAGCGTG \\
\hline VP2 C97S & Forward & 21-mer & ATCTGCAACAGCGGACAATTC \\
\hline VP2 C97S & Reverse & 24-mer & ATTGTCCGCTGTTGCAGATCTTAG \\
\hline VP2 C95S/C97S & Forward & 28-mer & CGCTAAGATCAGCAACAGCGGACAATTC \\
\hline VP2 C95S/C97S & Reverse & 28-mer & ATTGTCCGCTGTTGCTGATCTTAGCGTG \\
\hline
\end{tabular}


Dulbecco's minimal essential medium (DMEM) (Invitrogen, USA) supplemented with $10 \%$ (v/v) FBS, 100 units/ $\mathrm{ml}$ penicillin, and $100 \mu \mathrm{g} / \mathrm{ml}$ streptomycin. In order to determine the localization of VP2-GFP and the various mutant proteins, pcDNA3.1 VP2-GFP and the various mutant plasmids were transfected into $\mathrm{HeLa}$ and $\mathrm{CHO}$ cells using TurboFect ${ }^{\mathrm{TM}}$ (Fermentas, Canada) by following the manufacturer's instructions. The plasmid of pAcGFP-N1 VP3-GFP was also transfected as a positive control for the LMB treatment assay. HeLa or CHO cells were seeded at a density of $2 \times 10^{4}$ or $8 \times 10^{4}$ cells per well in 24 well culture plates. Forty-eight hours after transfection, aliquots of the cells, underwent replacement with fresh medium containing $20 \mathrm{ng} / \mathrm{ml} \mathrm{LMB} \mathrm{(+)}$ (Calbiochem, Germany) [27,28] or phosphate-buffered saline (PBS) buffer as the LMB (-) control. This was in order to test the nuclear export signals of VP2 and VP3. All cells were fixed using 4\% paraformaldehyde solution. After washing three times with $1 \times$ PBS, the cells were incubated with $1 \times$ PBS containing $0.25 \%$ Triton X-100 for $10 \mathrm{~min}$ and stained with $1 \mu \mathrm{g} / \mathrm{ml}$ DAPI (Sigma, USA). GFP fluorescence and DAPI images were captured using a ZEISS AXIOVERT 200 microscope equipped with a 40 objective and an AxioCam HRm CCD camera. Image processing was done using Photoshop. The Alpha View ${ }^{\circledR}$ Software (Alpha Innotech Corporation, USA) was used to calculate the distribution of VP2-GFP and mutants across the cytosol and nucleus in the cells.

\section{Cell fractionations}

Chromatin fractions were prepared by minor modifications of a procedure described previously [42]. After transfection with VP2-GFP plasmid, the $\mathrm{CHO}$ cells were lysed in cytoskeleton (CSK) buffer containing 0.5\% Triton X-100, $1 \mathrm{mM}$ ATP, $1 \mathrm{mM}$ dithiothreitol, and protease inhibitors (Sigma, USA) for $30 \mathrm{~min}$ on ice. The protein extract was then centrifuged at $1500 \mathrm{~g}$ for $5 \mathrm{~min}$ at $4^{\circ} \mathrm{C}$. The supernatant was collected and labeled as the soluble fraction $(\mathrm{S})$. The pellet which was chromatin $(\mathrm{C})$ was washed once with CSK buffer for $5 \mathrm{~min}$ on ice, then centrifuged at $1500 \mathrm{~g}$ for $5 \mathrm{~min}$ at $4^{\circ} \mathrm{C}$, and resuspended in SDS sample buffer. For micrococcal nuclease (MNase) (Fermentas, Canada) or $\mathrm{NaCl}$ treatment, the chromatin was resuspended in $100 \mu \mathrm{l}$ of CSK buffer supplemented with $0.1 \mathrm{M}, 0.3 \mathrm{M}$ of $\mathrm{NaCl}$ or $0 \mathrm{U}(-)$ and $150 \mathrm{U}(+)$ MNase with $2 \mathrm{mM} \mathrm{CaCl}_{2}$ and incubated for $30 \mathrm{~min}$ at $37^{\circ} \mathrm{C}$. After incubation, the chromatin and soluble supernatant were separated by centrifugation. Chromatin and soluble solutions were resuspended and boiled in SDS sample buffer for immunoblotting.

\section{Co-immunoprecipitation}

After transient transfection with pcDNA 3.1 Flag-VP2GFP and the various mutants, $\mathrm{CHO}$ cells were harvested and lysed using Nonidet P-40 (NP-40) lysis buffer (10 mM HEPES, $\mathrm{pH}$ 8.0, 0.2\% NP-40, $150 \mathrm{mM} \mathrm{NaCl}, 1 \mathrm{mM}$ EGTA, $5 \mathrm{mM} \mathrm{MgCl}_{2}$ and $10 \%$ glycerol) containing a cocktail of protease inhibitors (Sigma, USA). After 30 min on ice, the lysates were centrifuged at $13200 \mathrm{rpm}$ for $5 \mathrm{~min}$ at $4^{\circ} \mathrm{C}$. For co-immunoprecipitation, the 500 $\mu \mathrm{g} / \mathrm{ml}$ cell extracts were incubated with $2 \mu \mathrm{g}$ of antiFlag M2 beads (Sigma, USA) for $16 \mathrm{~h}$ at $4^{\circ} \mathrm{C}$. The immunoprecipitate was then washed three times with lysis buffer, resuspended in $20 \mu \mathrm{l}$ of SDS sample buffer, and the samples were heated for $5 \mathrm{~min}$ at $100^{\circ} \mathrm{C}$. The soluble proteins were resolved by SDS-PAGE for Western blotting.

\section{Additional material}

Additional file 1: The effect of LMB treatments on the various mutants of VP2. The truncated mutants of VP2 in Figure 3A were all treated with LMB (+) $(20 \mathrm{ng} / \mathrm{ml})$ and LMB (-) (PBS buffer) for $1 \mathrm{~h}$ at $37^{\circ} \mathrm{C}$. VP3-GFP is LMB sensitive and was used as a positive control. The distribution of GFP was monitored by fluorescence microscopy.

Additional file 2: Identification of protein-protein interactions with VP2 surveyed by co-immunoprecipitation. At $48 \mathrm{~h}$ post-transfection with plasmids encoding GFP (as the Control) or Flag-VP2-GFP (WT), cell lysates were immunoprecipitated by Flag $M 2$ beads and immunoblotted against VP2, MCM3, CDC7, SMC2, PCNA, and H2B antibodies. The nuclear extracts containing VP2-GFP are designated as N.

\section{Acknowledgements}

The authors would like to thank the Prof. Min-Ying Wang (the Graduate Institute of Biotechnology, National Chung Hsing University, Taichung, Taiwan) for kindly providing the pcDNA3.1-GFP plasmid. This research was supported by grants from the National Science Council (Grant nos. NSC-952313-B-039-004-; NSC-96-2313-B-276-001-MY3), China Medical University (CMU100-TS-05) as well as CMRPG860542 and CZRPG860563.

\section{Author details}

'Department of Medical Research, Chang Gung Memorial HospitalKaohsiung Medical Center, Chang Gung University College of Medicine, Kaohsiung, Taiwan. ${ }^{2}$ Department of Food Science, National Pingtung University of Science and Technology, Pingtung, Taiwan. ${ }^{3}$ Department of Veterinary Medicine, National Pingtung University of Science and Technology, Pingtung, Taiwan. ${ }^{4}$ Department of Medical Research, Tung's Taichung Metro Harbor Hospital, Taichung, Taiwan. ${ }^{5}$ Department of Safety, Health and Environmental Engineering, Mingchi University of Technology, Taipei, Taiwan. ${ }^{6}$ School of Chinese Pharmaceutical Science and Chinese Medicine Resources, China Medical University, Taichung, Taiwan.

\section{Authors' contributions}

JHC and MSL participated in the design and coordination of the study, carried out the study, performed the bioinformatics analysis, and wrote the manuscript. SCS and YYL participated in the design of the primers, experiments for clone and wrote the manuscript. MSL and WHS participated in experiments for clone. HJC participated in the bioinformatics analysis. All authors read and approved the final manuscript. 


\section{Competing interests}

MSL, JHC and YYL are inventors on a patent submission entitled: A method for Protein Translocating into Nucleus Using A Specific Peptide Sequence for. The other authors declare no competing interests.

Received: 21 August 2011 Accepted: 7 February 2012

Published: 7 February 2012

\section{References}

1. Yuasa NTT, Yoshida I: Isolation and some characteristics of an agent inducing anemia in chicks. Avian Dis 1979, 23:366-385.

2. Noteborn MH: Chicken anemia virus induced apoptosis: underlying molecular mechanisms. Vet Microbiol 2004, 98:89-94.

3. McNulty MS, Connor TJ, McNeilly F, Spackman D: Chicken anemia agent in the United States: isolation of the virus and detection of antibody in broiler breeder flocks. Avian Dis 1989, 33:691-694.

4. Yuasa NNT, Furuta K, Yoshida I: Maternal antibody and its effect on the susceptibility of chicks to chicken anemia agent. Avian Dis 1980, 24:197-201.

5. Lucio B, Schat KA, Shivaprasad HL: Identification of the chicken anemia agent, reproduction of the disease, and serological survey in the United States. Avian Dis 1990, 34:146-153.

6. Yuasa N, Imai K, Watanabe K, Saito F, Abe M, Komi K: Aetiological examination of an outbreak of haemorrhagic syndrome in a broiler flock in Japan. Avian Pathol 1987, 16:521-526.

7. Hsu JP, Lee ML, Lu YP, Hung HT, Hung HH, Chein MS: Chicken infectious anemia in layer. J Chin Soc Vet Sci 2002, 28:153-160.

8. Schat KA: Chicken anemia virus. Curr Top Microbiol Immunol 2009, 331:151-183.

9. Lu YS, Tsai HJ, Kwang MJ, Tseng CS: Chicken infectious anemia in Taiwan: virus isolation and antibody survey. Exp Rep TPRIAH 1993, 29:81-89.

10. Meehan BM, Todd D, Creelan JL, Earle JA, Hoey EM, McNulty MS: Characterization of viral DNAs from cells infected with chicken anaemia agent: sequence analysis of the cloned replicative form and transfection capabilities of cloned genome fragments. Arch Virol 1992, 124:301-319.

11. Claessens JA, Schrier CC, Mockett AP, Jagt EH, Sondermeijer PJ: Molecular cloning and sequence analysis of the genome of chicken anaemia agent. J Gen Virol 1991, 72(Pt 8):2003-2006.

12. Ducatez MF, Owoade AA, Abiola JO, Muller CP: Molecular epidemiology of chicken anemia virus in Nigeria. Arch Virol 2006, 151:97-111.

13. Phenix KV, Meehan BM, Todd D, McNulty MS: Transcriptional analysis and genome expression of chicken anaemia virus. J Gen Virol 1994, 75(Pt 4):905-909.

14. Noteborn MH, Kranenburg O, Zantema A, Koch G, de Boer GF, van der Eb AJ: Transcription of the chicken anemia virus (CAV) genome and synthesis of its 52-kDa protein. Gene 1992, 118:267-271.

15. Pallister J, Fahey KJ, Sheppard M: Cloning and sequencing of the chicken anaemia virus (CAV) ORF-3 gene, and the development of an ELISA for the detection of serum antibody to CAV. Vet Microbiol 1994, 39:167-178.

16. Douglas AJ, Phenix K, Mawhinney KA, Todd D, Mackie DP, Curran WL: Identification of a $24 \mathrm{kDa}$ protein expressed by chicken anaemia virus. $J$ Gen Virol 1995, 76(Pt 7):1557-1562.

17. Peters MA, Jackson DC, Crabb BS, Browning GF: Chicken anemia virus VP2 is a novel dual specificity protein phosphatase. J Biol Chem 2002, 277:39566-39573.

18. Mendez J, Stillman B: Chromatin association of human origin recognition complex, cdc6, and minichromosome maintenance proteins during the cell cycle: assembly of prereplication complexes in late mitosis. Mol Cell Biol 2000, 20:8602-8612.

19. Noteborn MH, Todd D, Verschueren CA, de Gauw HW, Curran WL, Veldkamp S, Douglas AJ, McNulty MS, van Der EA, Koch G: A single chicken anemia virus protein induces apoptosis. J Virol 1994, 68:346-351

20. Kamada K, Kuroishi A, Kamahora T, Kabat P, Yamaguchi S, Hino S: Spliced mRNAs detected during the life cycle of Chicken anemia virus. J Gen Virol 2006, 87:2227-2233.

21. Koch G, van Roozelaar DJ, Verschueren CA, van der Eb AJ, Noteborn MH: Immunogenic and protective properties of chicken anaemia virus proteins expressed by baculovirus. Vaccine 1995, 13:763-770.

22. Noteborn MH, Verschueren CA, Koch G, Van der Eb AJ: Simultaneous expression of recombinant baculovirus-encoded chicken anaemia virus
(CAV) proteins VP1 and VP2 is required for formation of the CAV-specific neutralizing epitope. J Gen Virol 1998, 79(Pt 12):3073-3077.

23. Noteborn MH, Verschueren CA, van Ormondt $\mathrm{H}$, van der Eb AJ: Chicken anemia virus strains with a mutated enhancer/promoter region share reduced virus spread and cytopathogenicity. Gene 1998, 223:165-172.

24. Adair BM: Immunopathogenesis of chicken anemia virus infection. Dev Comp Immunol 2000, 24:247-255.

25. Lacorte C, Lohuis H, Goldbach R, Prins M: Assessing the expression of chicken anemia virus proteins in plants. Virus Res 2007, 129:80-86.

26. Nigg EA: Nucleocytoplasmic transport: signals, mechanisms and regulation. Nature 1997, 386:779-787.

27. Davis M, Hatzubai A, Andersen JS, Ben-Shushan E, Fisher GZ, Yaron A, Bauskin A, Mercurio F, Mann M, Ben-Neriah Y: Pseudosubstrate regulation of the SCF(beta-TrCP) ubiquitin ligase by hnRNP-U. Genes Dev 2002, 16:439-451.

28. Kosugi S, Hasebe M, Tomita M, Yanagawa H: Nuclear export signal consensus sequences defined using a localization-based yeast selection system. Traffic 2008, 9:2053-2062.

29. Poon IK, Oro C, Dias MM, Zhang J, Jans DA: Apoptin nuclear accumulation is modulated by a CRM1-recognized nuclear export signal that is active in normal but not in tumor cells. Cancer Res 2005, 65:7059-7064.

30. Wang QM, Fan GC, Chen JZ, Chen HP, He FC: A putative NES mediates cytoplasmic localization of Apoptin in normal cells. Acta Biochim Biophys Sin (Shanghai) 2004, 36:817-823.

31. Peters MA, Jackson DC, Crabb BS, Browning GF: Mutation of chicken anemia virus VP2 differentially affects serine/threonine and tyrosine protein phosphatase activities. J Gen Virol 2005, 86:623-630.

32. Todd D, McNulty MS, Adair BM, Allan GM: Animal circoviruses. Adv Virus Res 2001, 57:1-70.

33. Leliveld SR, Dame RT, Rohn JL, Noteborn MHM, Abrahams JP: Apoptin's functional $\mathrm{N}$ - and C-termini independently bind DNA. FEBS Letters 2004, 557:155-158.

34. Edwards MC, Tutter AV, Cvetic C, Gilbert CH, Prokhorova TA, Walter JC: MCM2-7 complexes bind chromatin in a distributed pattern surrounding the origin recognition complex in Xenopus egg extracts. J Biol Chem 2002, 277:33049-33057.

35. Rowles A, Blow Jj: Chromatin proteins involved in the initiation of DNA replication. Curr Opin Genet Dev 1997, 7:152-157.

36. Mankertz A, Hillenbrand B: Replication of porcine circovirus type 1 requires two proteins encoded by the viral rep gene. Virology 2001, 279:429-438.

37. Lee MS, Lien YY, Feng SH, Huang RL, Tsai MC, Chang WT, Chen HJ: Production of chicken anemia virus (CAV) VP1 and VP2 protein expressed by recombinant Escherichia coli. Proc Biochem 2009, 44:390-395.

38. Huang CH, Lai GH, Lee MS, Lin WH, Lien YY, Hsueh SC, Kao JY, Chang WT, Lu TC, Lin WN, Chen HJ: Development and evaluation of a loopmediated isothermal amplification assay for rapid detection of chicken anaemia virus. J Appl Microbiol 2010, 108:917-924.

39. Ia Cour T, Kiemer L, Molgaard A, Gupta R, Skriver K, Brunak S: Analysis and prediction of leucine-rich nuclear export signals. Protein Eng Des Sel 2004, 17:527-536.

40. Horton P, Park KJ, Obayashi T, Fujita N, Harada H, Adams-Collier CJ, Nakai K: WoLF PSORT: protein localization predictor. Nucleic Acids Res 2007, 35: W585-587.

41. Nguyen Ba AN, Pogoutse A, Provart N, Moses AM: NLStradamus: a simple Hidden Markov Model for nuclear localization signal prediction. BMC Bioinformatics 2009, 10:202.

42. Deng M, Li F, Ballif BA, Li S, Chen X, Guo L, Ye X: Identification and functional analysis of a novel cyclin e/cdk2 substrate ankrd17. J Biol Chem 2009, 284:7875-7888.

doi:10.1186/1746-6148-8-15

Cite this article as: Cheng et al:: Identification of the NLS and NES motifs of VP2 from chicken anemia virus and the interaction of VP2 with mini-chromosome maintenance protein 3. BMC Veterinary Research 2012 8:15. 